\title{
MULTIDOMAIN SINGULAR BOUNDARY METHOD FOR 2D LAMINAR VISCOUS FLOW
}

\author{
JURAJ MUŽÍIK \& ROMAN BULKO \\ Department of Geotechnics, Faculty of Civil Engineering, University of Zilina, Slovakia
}

\begin{abstract}
In this paper, a numerical algorithm is developed for the solution of two-dimensional isothermal laminar viscous flow. The proposed numerical method implementation, which is quadrature-free, is based on the stream-velocity formulation of Navier-Stokes equations, the method of the particular solutions and the singular boundary method (SBM). The steady stream-velocity formulation of NS equation is 4th order biharmonic non-homogeneous nonlinear equation type which is solved using the proposed method with the non-homogeneous and nonlinear terms approximated using the method of particular solutions with multiquadrics RBF function. The accuracy of the method is proven using 2D backwardfacing step and lid-driven cavity test cases.

Keywords: singular boundary method, dual reciprocity, Navier-Stokes equation, biharmonic equations.
\end{abstract}

\section{INTRODUCTION}

The numerical codes based on the theory of boundary integral equations are represented mainly by the boundary element method (BEM) [1], [2], that is one of the very important numerical methods used for scientific research and engineering practice.

However, the BEM is a very efficient numerical method from the mathematical and engineering point of view it brings some drawbacks caused mainly by time-consuming process of numerical integration. On the other side the boundary collocation methods, like the most-known method of fundamental solution (MFS) avoids the process of numerical integration and creation of a computational mesh in the sense of BEM. The singularity problem of the fundamental solution in MFS is overcome by construction of a fictitious boundary. The optimal position of the nodes that make up fictitious this boundary remains the open problem. To avoid the need for the fictitious boundary creation Sarler [5] proposed the modified method of fundamental solutions (MMFS). The MMFS uses the same set of boundary nodes as source and response nodes, but it requires a computationally intensive calculation of the characteristic matrix diagonal elements.

Chen et al. [6] and Chen and $\mathrm{Gu}$ [7] presented a numerical alternative to MMFS called a singular boundary method (SBM). Like MMFS, the SBM is using the nodes of regular boundary only and regularizes the singularities of fundamental solutions (diagonal elements of the characteristic matrix) using the values of so-called origin intensity factors (OIFs). There are several rigorous or empirical techniques used to determine the OIFs values, namely, inverse interpolation technique (IIT), subtracting and adding-back regularization and formulas [8], [9] based on empirical values. In this article, the regularized boundary integral equation is used to derive a formula for Dirichlet origin intensity factors.

This paper describes the possibility of the steady laminar isothermal flow solution, which is governed by the biharmonic (velocity-stream) form of Navier-Stokes (NS) equation using SBM with dual reciprocity (DR) scheme.

Section 2 of our paper introduces NS equations in velocity-stream form. Section 3 presents the basal concepts of the SBM and the connection with the DRM. Sections 4 and 5 are dedicated to two numerical examples and Section 6 contains conclusions. 


\section{GOVERNING EQUATIONS}

An unsteady incompressible flow is governed by Navier-Stokes equations, which can be written in the non-dimensional form using primitive variables as

$$
\begin{gathered}
\frac{\partial u}{\partial t}+u \frac{\partial u}{\partial x}+v \frac{\partial u}{\partial y}=-\frac{\partial p}{\partial x}+\frac{1}{R e}\left(\frac{\partial^{2} u}{\partial x^{2}}+\frac{\partial^{2} u}{\partial y^{2}}\right) \\
\frac{\partial v}{\partial t}+u \frac{\partial v}{\partial x}+v \frac{\partial v}{\partial y}=-\frac{\partial p}{\partial y}+\frac{1}{R e}\left(\frac{\partial^{2} v}{\partial x^{2}}+\frac{\partial^{2} v}{\partial y^{2}}\right) \\
\frac{\partial v}{\partial x}+\frac{\partial v}{\partial y}=0
\end{gathered}
$$

where $u$ and $v$ are horizontal and vertical velocity components respectively, $p$ represents the pressure, $R e$ is the non-dimensional Reynolds number and $x, y$ are the spatial coordinates and $t$ is the time.

Though this formulation accurately represents the fluid flow, its direct solution has been difficult to obtain due to the mixed formulation in eqns (1), (2) and lack of the pressure development equation. To avoid the direct solution of the pressure field, a formulation using stream function and vorticity has been used as an alternative for several decades [10]. This formulation introduces the stream function $\psi$ and vorticity $\omega$ :

$$
\begin{gathered}
\frac{\partial^{2} \psi}{\partial x^{2}}+\frac{\partial^{2} \psi}{\partial y^{2}}=-\omega \\
\frac{\partial \omega}{\partial t}+u \frac{\partial \omega}{\partial x}+v \frac{\partial \omega}{\partial y}=\frac{1}{R e}\left(\frac{\partial^{2} \omega}{\partial x^{2}}+\frac{\partial^{2} \omega}{\partial y^{2}}\right) .
\end{gathered}
$$

This formulation has been very successful and has been used by many researchers over the past several decades to test new methods for the numerical solutions of a variety of fluid flow problems. Typical difficulty with this formulation consists of the specification of vorticity values at the no-slip boundaries; the vorticity $\omega$ is defined through the Poisson eqn (4) which needs to be solved discretely on the boundaries so that boundary values of the vorticity can be specified for the vorticity transport eqn (5) when this formulation is utilized. However, the values of vorticity $\omega$ on the boundaries are generally unspecified and one must carry out a variety of numerical approximations in order to specify the boundary values of vorticity [10].

To avoid the process of determination of boundary vorticity values the vorticity can be eliminated from the eqns (4), (5) at the price of 4th order non-linear, non-homogeneous, biharmonic partial differential eqn (6) [11]:

$$
\frac{\partial^{4} \psi}{\partial x^{4}}+2 \frac{\partial^{4} \psi}{\partial x^{2} \partial y^{2}}+\frac{\partial^{4} \psi}{\partial y^{4}}-\operatorname{Re}\left[u\left(\frac{\partial^{3} \psi}{\partial x^{3}}+\frac{\partial^{3} \psi}{\partial x \partial y^{2}}\right)+v\left(\frac{\partial^{3} \psi}{\partial y^{3}}+\frac{\partial^{3} \psi}{\partial x^{2} \partial y}\right)\right]=0,
$$

where $\psi$ represents stream function, $u$ and $v$ are velocity components that can be expressed in the terms of stream function as

$$
u=\frac{\partial \psi}{\partial y}, \quad v=-\frac{\partial \psi}{\partial x} .
$$

The boundary conditions of eqn (6) are divided into two basic groups

- two boundary conditions of the Dirichlet type, where the value of variable $\psi$ or its Laplacian $\Delta \psi$ on the part of boundary $\Gamma_{1}$ or $\Gamma_{3}$ is given, i.e.

$$
\psi=\mathrm{U}_{0}(\mathbf{x}) \mathbf{x} \in \Gamma_{1}, \Delta \psi=\mathrm{V}_{0}(\mathbf{x}) \mathbf{x} \in \Gamma_{3}
$$


- two boundary conditions of the Neumann type, where the value of the normal derivative of stream function or its Laplacian on the boundary $\Gamma_{2}$ or $\Gamma_{4}$ is given, i.e.

$$
\frac{\partial \psi}{\partial \mathrm{n}}=\mathrm{q}_{0}(\mathbf{x}) \mathbf{x} \in \Gamma_{2}, \frac{\partial \Delta \psi}{\partial \mathrm{n}}=\mathrm{p}_{0}(\mathbf{x}) \mathbf{x} \in \Gamma_{4}
$$

where $\mathrm{n}$ is the outward normal to the boundary $\Gamma_{2}$ or $\Gamma_{4}$.

For the numerical solution of the biharmonic eqn (6) the two boundary conditions need to be specified to obtain a solution [11].

\section{SINGULAR BOUNDARY METHOD}

The principle of the MFS is up to some level used by the singular boundary method (SBM) The main difference and superiority of SBM over MFS is no need of fictitious boundary. The SBM uses the same set of nodes as source and response points positioned on regular the boundary $\Gamma$. The solution of the homogeneous biharmonic equation is approximated by the linear combination of Laplace (11) and biharmonic (10) fundamental solutions (see eqn (12)) [12]

$$
\begin{gathered}
G_{0}=-\frac{\|\mathbf{x}-\mathbf{s}\|^{2}}{2 \pi}(\ln \|\mathbf{x}-\mathbf{s}\|-1), \\
G_{1}=-\frac{1}{2 \pi} \ln \|\mathbf{x}-\mathbf{s}\|, \\
\psi_{h}(\mathbf{x})=\sum_{j=1}^{N} a_{j} G_{0}\left(\mathbf{x}, \mathbf{s}_{\mathbf{j}}\right)+\sum_{j=1}^{N} b_{j} G_{1}\left(\mathbf{x}, \mathbf{s}_{\mathbf{j}}\right), \quad \mathbf{x} \in \Omega \cup \Gamma .
\end{gathered}
$$

The diagonal term that arises when the source and response nodes are at the same position [7]-[9] can't be evaluated directly and needs to be determined using one of the aforementioned procedures and this process represents the crucial part of the SBM. To elaborate the solution (12) the singular terms that cannot be directly computed are moved outside the sum

$$
\psi_{h}\left(\mathbf{x}_{i}\right)=\sum_{j=1, j \neq i}^{N} a_{j} G_{0}\left(\mathbf{x}_{i}, \mathbf{s}_{j}\right)+a_{i} U_{i i}^{0}+\sum_{j=1, j \neq i}^{N} b_{j} G_{1}\left(\mathbf{x}_{i}, \mathbf{s}_{j}\right)+b_{i} U_{i i}^{1}, \quad \mathbf{x}_{i} \in \Gamma,
$$

and similarly, for the boundary normal derivative at location $\mathbf{x}_{i}$

$$
\frac{\partial \psi_{h}\left(\mathbf{x}_{i}\right)}{\partial n_{x_{i}}}=\sum_{j=1, j \neq i}^{N} a_{j} \frac{\partial G_{0}\left(\mathbf{x}_{i}, \mathbf{s}_{j}\right)}{\partial n_{x_{i}}}+a_{i} Q_{i i}^{0}+\sum_{j=1, j \neq i}^{N} b_{j} \frac{\partial G_{1}\left(\mathbf{x}_{i}, \mathbf{s}_{j}\right)}{\partial n_{x_{i}}}+b_{i} Q_{i i}^{1}, \quad \mathbf{x}_{i} \in \Gamma,
$$

the unknown coefficient $U_{i i}^{0}, Q_{i i}^{0}, U_{i i}^{1}, Q_{i i}^{1}$ are defined as OIFs. OIFs for a biharmonic fundamental solution can be handled very easy using limit to eliminate the singularity as the collocation point approaches the source point. So, for the OIFs that arises from biharmonic fundamental solution one can write

$$
\begin{gathered}
U_{i i}^{0}=\lim _{\mathbf{x}_{i} \rightarrow \mathbf{s}_{i}} G_{0}\left(\mathbf{x}_{i}, \mathbf{s}_{i}\right)=0, \\
Q_{i i}^{0}=\lim _{\mathbf{x}_{i} \rightarrow \mathbf{s}_{i}} \frac{\partial G_{0}\left(\mathbf{x}_{i}, \mathbf{s}_{i}\right)}{\partial n_{\mathbf{x}_{i}}}=0 .
\end{gathered}
$$

The OIFs associated with the Laplace fundamental solution can be determined using subtracting and adding-back technique for the normal derivative (Neumann) boundary condition [7], [13]

$$
Q_{i i}^{1}=-\frac{1}{L_{i}} \sum_{j=1, j \neq i}^{N} L_{j} \frac{\partial G_{1}\left(\mathbf{x}_{i}, \mathbf{s}_{j}\right)}{\partial n_{\mathbf{s}_{j}}}
$$


where $L_{j}$ is the length of the appropriate part of boundary around point $\mathbf{x}_{i}$ (see Fig. 1). The value of the OIF for prescribed solution value (Dirichlet BC) on the boundary, the formula based on the regularized boundary integral equation is used [13]

$$
U_{i i}^{1}=-\frac{1}{L_{i}} \sum_{j=1, j \neq i}^{N} L_{j}\left[\mathbf{n}_{\mathbf{s}_{i}} \cdot \mathbf{n}_{\mathbf{x}_{j}} G_{1}\left(\mathbf{x}_{i}, \mathbf{s}_{j}\right)-\mathbf{n}_{\mathbf{s}_{i}} \cdot\left(\mathbf{x}_{j}-\mathbf{s}_{i}\right) \frac{\partial G_{1}\left(\mathbf{x}_{j}, \mathbf{s}_{i}\right)}{\partial n_{\mathbf{x}_{j}}}\right] .
$$

\subsection{Dual reciprocity method}

The biharmonic (stream - velocity) form of the Navier-Stokes equation represents nonlinear, non-homogeneous boundary value problem. The governing eqn (6) can be rearranged

$$
\frac{\partial^{4} \psi}{\partial x^{4}}+2 \frac{\partial^{4} \psi}{\partial x^{2} \partial y^{2}}+\frac{\partial^{4} \psi}{\partial y^{4}}=\Delta^{2} \psi=\operatorname{Re}\left[u\left(\frac{\partial^{3} \psi}{\partial x^{3}}+\frac{\partial^{3} \psi}{\partial x \partial y^{2}}\right)+v\left(\frac{\partial^{3} \psi}{\partial y^{3}}+\frac{\partial^{3} \psi}{\partial x^{2} \partial y}\right)\right] .
$$

The solution of (19) can now be defined as the sum

$$
\psi=\psi_{h}+\psi_{p},
$$

where $\psi \mathrm{h}$ is a solution that satisfies the homogeneous biharmonic equation and prescribed boundary conditions. The $\psi \mathrm{p}$ represents a particular solution of the non-homogeneous eqn (19). The homogeneous part of the solution was calculated using the singular boundary method (SBM) and the particular part of the solution is obtained adopting dual reciprocity method. The particular solution $\psi \mathrm{p}$ is approximated as a linear combination of radial basis (RBF) kernels as

$$
\psi_{p}=\sum_{j=1}^{M} \beta_{j} \Phi\left(r_{j}\right),
$$

where $\Phi\left(r_{j}\right)$ are radial basis functions, $\beta_{j}$ are constants to be determined and $M$ is the number of the internal points. The function $\Phi\left(r_{j}\right)$ represents a solution of the following equation

$$
\Delta^{2} \Phi\left(r_{j}\right)=\varphi\left(r_{j}\right),
$$

where $\varphi\left(r_{j}\right)$ are also radial basis kernels. The multiquadrics RBF (MQ see Table 1) have been tested for computations presented in this paper. Other appropriate possibilities can be found in [2], [13].

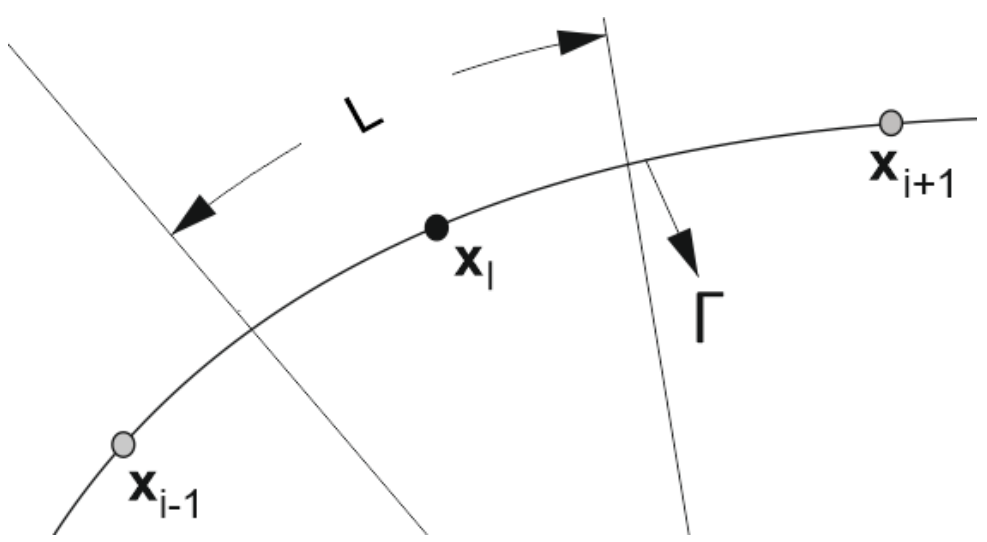

Figure 1: Part of boundary $L$ around point $\mathbf{x}_{i}$. 
Table 1: Integrated MQ radial basis functions tested in this paper.

\begin{tabular}{|c|c|c|}
\hline$\varphi(r)$ & $\Delta \Phi(r)$ & $\Phi(r)$ \\
\hline$\sqrt{r^{2}+c^{2}}$ & $\frac{1}{9}\left(r^{2}+4 c^{2}\right) \sqrt{r^{2}+c^{2}}-\frac{c^{3}}{3} \ln \left(c \sqrt{r^{2}+c^{2}}+c^{2}\right)$ & $\frac{1}{900}\left(\begin{array}{c}75 c^{3} r^{2}-61 c^{4} \sqrt{r^{2}+c^{2}}+48 c^{2} r^{2} \sqrt{r^{2}+c^{2}} \\
+4 r^{4} \sqrt{r^{2}+c^{2}}+\left(30 c^{5}-75 c^{3} r^{2}\right) \ln \left(c+\sqrt{r^{2}+c^{2}}\right)\end{array}\right)$ \\
\hline
\end{tabular}

According to the above definitions and the eqn (19), we can write

$$
\sum_{j=1}^{M} \beta_{j} \varphi\left(r_{j}\right)=\operatorname{Re}\left[\frac{\partial \psi}{\partial y}\left(\frac{\partial^{3} \psi}{\partial x^{3}}+\frac{\partial^{3} \psi}{\partial x \partial y^{2}}\right)-\frac{\partial \psi}{\partial x}\left(\frac{\partial^{3} \psi}{\partial y^{3}}+\frac{\partial^{3} \psi}{\partial x^{2} \partial y}\right)\right],
$$

because the nonlinear character of eqn (17) iterative procedure is adopted to solve eqn (23) while the terms on the right-hand side of eqn (23) will be evaluated using MQ (see Table 1) interpolation functions with coefficients $\beta$ and fundamental solutions with coefficients $a$ and $b$ knew from the previous iteration. The explicit iterative form of eqn (23) one can write as

$$
\begin{aligned}
& \sum_{\mathrm{j}=1}^{\mathrm{M}} \beta_{\mathrm{j}}^{\mathrm{n}+1} \varphi\left(\mathrm{x}, \mathrm{s}_{\mathrm{j}}\right)=\operatorname{Re}\left[\left(\sum_{\mathrm{j}=1}^{\mathrm{M}} \beta_{\mathrm{j}}^{\mathrm{n}} \frac{\partial \Phi\left(\mathrm{x}, \mathrm{s}_{\mathrm{j}}\right)}{\partial \mathrm{y}}+\sum_{\mathrm{i}=1}^{\mathrm{N}} \mathrm{a}_{\mathrm{i}}^{\mathrm{n}} \frac{\partial \mathrm{G} 0\left(\mathrm{x}, \mathrm{s}_{\mathrm{i}}\right)}{\partial \mathrm{y}}+\right.\right. \\
& \left.\sum_{\mathrm{i}=1}^{\mathrm{N}} \mathrm{b}_{\mathrm{i}}^{\mathrm{n}} \frac{\partial \mathrm{G} 1\left(\mathrm{x}, \mathrm{s}_{\mathrm{i}}\right)}{\partial \mathrm{y}}\right)\left(\sum_{\mathrm{j}=1}^{\mathrm{M}} \beta_{\mathrm{j}}^{\mathrm{n}}\left(\frac{\partial^{3} \Phi\left(\mathrm{x}, \mathrm{s}_{\mathrm{j}}\right)}{\partial \mathrm{x}^{3}}+\frac{\partial^{3} \Phi\left(\mathrm{x}, \mathrm{s}_{\mathrm{j}}\right)}{\partial \mathrm{x} \partial \mathrm{y}^{2}}\right)+\sum_{\mathrm{i}=1}^{\mathrm{N}} \mathrm{a}_{\mathrm{i}}^{\mathrm{n}}\left(\frac{\partial^{3} \mathrm{G} 0\left(\mathrm{x}, \mathrm{s}_{\mathrm{i}}\right)}{\partial \mathrm{x}^{3}}+\frac{\partial^{3} \mathrm{G} 0\left(\mathrm{x}, \mathrm{s}_{\mathrm{i}}\right)}{\partial \mathrm{x} \partial \mathrm{y}^{2}}\right)+\right. \\
& \left.\sum_{\mathrm{i}=1}^{\mathrm{N}} \mathrm{b}_{\mathrm{i}}^{\mathrm{n}}\left(\frac{\partial^{3} \mathrm{G} 1\left(\mathrm{x}, \mathrm{s}_{\mathrm{i}}\right)}{\partial \mathrm{x}^{3}}+\frac{\partial^{3} \mathrm{G} 1\left(\mathrm{x}, \mathrm{s}_{\mathrm{i}}\right)}{\partial \mathrm{x} \partial \mathrm{y}^{2}}\right)\right)-\left(\sum_{\mathrm{j}=1}^{\mathrm{M}} \beta_{\mathrm{j}}^{\mathrm{n}} \frac{\partial \Phi\left(\mathrm{x}, \mathrm{s}_{\mathrm{j}}\right)}{\partial \mathrm{x}}+\sum_{\mathrm{i}=1}^{\mathrm{N}} \mathrm{a}_{\mathrm{i}}^{\mathrm{n}} \frac{\partial \mathrm{G} 0\left(\mathrm{x}, \mathrm{s}_{\mathrm{i}}\right)}{\partial \mathrm{x}}+\right. \\
& \left.\sum_{\mathrm{i}=1}^{\mathrm{N}} \mathrm{b}_{\mathrm{i}}^{\mathrm{n}} \frac{\partial \mathrm{G} 1\left(\mathrm{x}, \mathrm{s}_{\mathrm{i}}\right)}{\partial \mathrm{x}}\right)\left(\sum_{\mathrm{j}=1}^{\mathrm{M}} \beta_{\mathrm{j}}^{\mathrm{n}}\left(\frac{\partial^{3} \Phi\left(\mathrm{x}, \mathrm{s}_{\mathrm{j}}\right)}{\partial \mathrm{y}^{3}}+\frac{\partial^{3} \Phi\left(\mathrm{x}, \mathrm{s}_{\mathrm{j}}\right)}{\partial \mathrm{x}^{2} \partial \mathrm{y}}\right)+\sum_{\mathrm{i}=1}^{\mathrm{N}} \mathrm{a}_{\mathrm{i}}^{\mathrm{n}}\left(\frac{\partial^{3} \mathrm{G} 0\left(\mathrm{x}, \mathrm{s}_{\mathrm{i}}\right)}{\partial \mathrm{y}^{3}}+\frac{\partial^{3} \mathrm{G} 0\left(\mathrm{x}, \mathrm{s}_{\mathrm{i}}\right)}{\partial \mathrm{x}^{2} \partial \mathrm{y}}\right)+\right. \\
& \left.\left.\sum_{\mathrm{i}=1}^{\mathrm{N}} \mathrm{b}_{\mathrm{i}}^{\mathrm{n}}\left(\frac{\partial^{3} \mathrm{G} 0\left(\mathrm{x}, \mathrm{s}_{\mathrm{i}}\right)}{\partial \mathrm{y}^{3}}+\frac{\partial^{3} \mathrm{G} 0\left(\mathrm{x}, \mathrm{s}_{\mathrm{i}}\right)}{\partial \mathrm{x}^{2} \partial \mathrm{y}}\right)\right)\right]
\end{aligned}
$$

Eqn (24) will be formed for every internal node ( $M$ - the number of internal nodes) which gives $M$ equations. To close the linear system, we must impose $2 N$ boundary condition values in all boundary nodes (two for each node). These $2 \mathrm{~N}$ equations are obtained imposing the first type of Dirichlet and Neumann boundary conditions

$$
\begin{gathered}
\sum_{i=1}^{N} a_{i}^{n+1} G 0\left(\mathbf{x}, \mathbf{s}_{i}\right)+\sum_{i=1}^{N} b_{i}^{n+1} G 1\left(\mathbf{x}, \mathbf{s}_{i}\right)+\sum_{j=1}^{M} \beta_{j}^{n+1} \Phi\left(\mathbf{x}, \mathbf{s}_{i}\right)=\bar{\psi}, \\
\sum_{i=1}^{N} a_{i} \frac{\partial G 0\left(\mathbf{x}, \mathbf{s}_{i}\right)}{\partial n}+\sum_{i=1}^{N} b_{i} \frac{\partial G 1\left(\mathbf{x}, \mathbf{s}_{i}\right)}{\partial n}+\sum_{j=1}^{M} \beta_{j} \frac{\partial \Phi\left(\mathbf{x}, \mathbf{s}_{i}\right)}{\partial n}=\bar{q},
\end{gathered}
$$

The closed system of eqns (24)-(26) is then iteratively solved until the overall difference between two successive steps is smaller than $\varepsilon=1 \times 10^{-5}$.

The DRM technique is applied to approximate nonlinear and non-homogeneous terms of eqn (19) over the whole computational domain. However, as has been remarked by several researchers, the DRM yields accurate stable solutions for a Reynolds number up to 200 .

This limitation shows that special domain treatments are required to obtain the solution for higher Reynolds numbers [14].

In the BEM focused articles, one can often find references to the division of computational domain into smaller regions - sub-domains [14]. When the convection effect becomes stronger, the sub-domain technique offers the possibility of emulating a computational mesh in homogeneous medium by dividing it into sub-domains and then applying the complete SBM with DRM to each of these sub-domains. Such a method used to be called Multiple Domain Singular Boundary Method with Dual Reciprocity - MD-SBMDR.

The detail of single simple square domain is shown in Fig. 2 with the detail of global computational domain "mesh". 

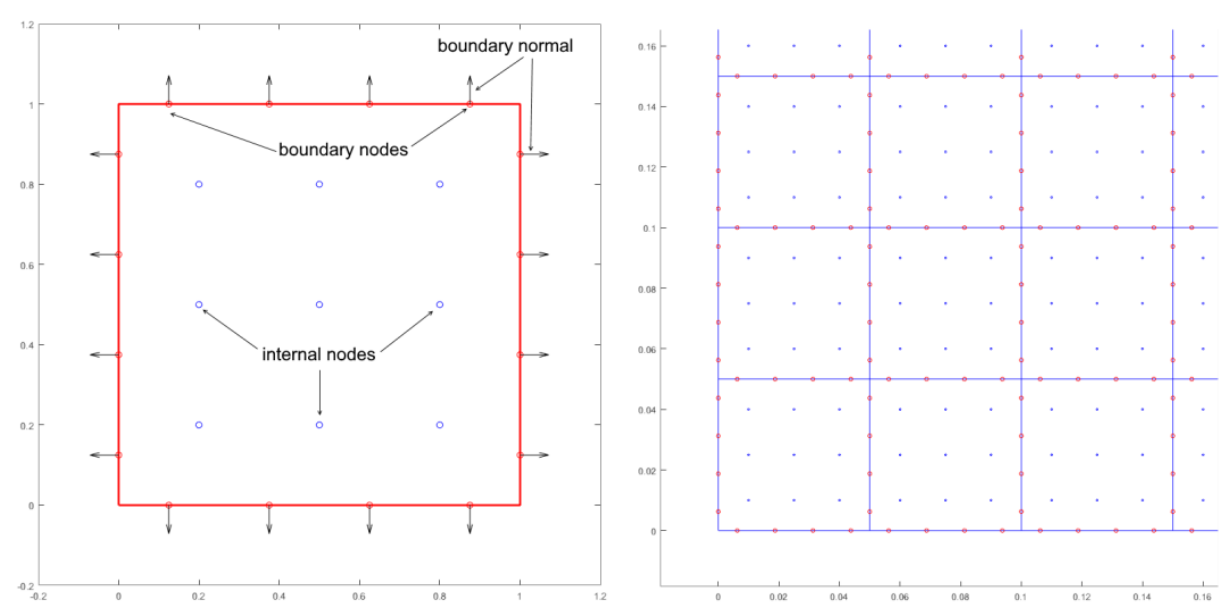

Figure 2: The sub-domain used in the MD-SBMDR formulation and "mesh" of multiple sub-domains covering the global computational domain.

\section{SIMULATION OF THE LID-DRIVEN CAVITY PROBLEM}

The standard benchmark problem for the numerical solver of incompressible, isothermal and laminar flow based on the Navier-Stokes equation is Lid-driven cavity flow. The top boundary of the cavity imposes a generation of the velocity $u=1$ to the flow moving the top lid, and no-slip impermeable boundary conditions are prescribed for the bottom, left and right wall. In a case of the stream function, the following boundary conditions are used

$$
\begin{gathered}
\psi(0, \mathrm{y})=\psi(1, \mathrm{y})=\psi(\mathrm{x}, 0)=\psi(\mathrm{x}, 1)=0, \\
\frac{\partial \psi(0, y)}{\partial n_{\text {left }}}=\frac{\partial \psi(1, y)}{\partial n_{\text {right }}}=\frac{\partial \psi(x, 0)}{\partial n_{\text {bottom }}}=0, \\
\frac{\partial \psi(x, 1)}{\partial n_{\text {top }}}=1 .
\end{gathered}
$$

The geometrical configuration and prescribed boundary conditions are shown in Fig. 3. The challenging aspect of this benchmark problem lies in the presence of singularities of pressure and velocity field at the two upper corners of the cavity. Therefore, the size of computational sub-domains used decreases as their position is close to these corners (see Fig. 3) to capture high stream function gradients.

The steady solution using the MD-SBMDR is reached when the difference between two consecutive time steps (in the sense of the infinity norm) is lower than the prescribed, $\varepsilon_{t o l}=$ $1 \times 10^{-6}$ is used for presented numerical computations.

The solutions obtained by the MD-SBMDR are compared with the solution presented in [15] for the Reynolds number $\mathrm{Re}=1000$; the vertical velocity components along a horizontal line and the horizontal velocity components along a vertical line through the center of the cavity are plotted in Fig. 4. Figs 5 and 6 present stream-function $\psi$ and pressure contours for Reynolds numbers $\mathrm{Re}=400$ and 1000 . 

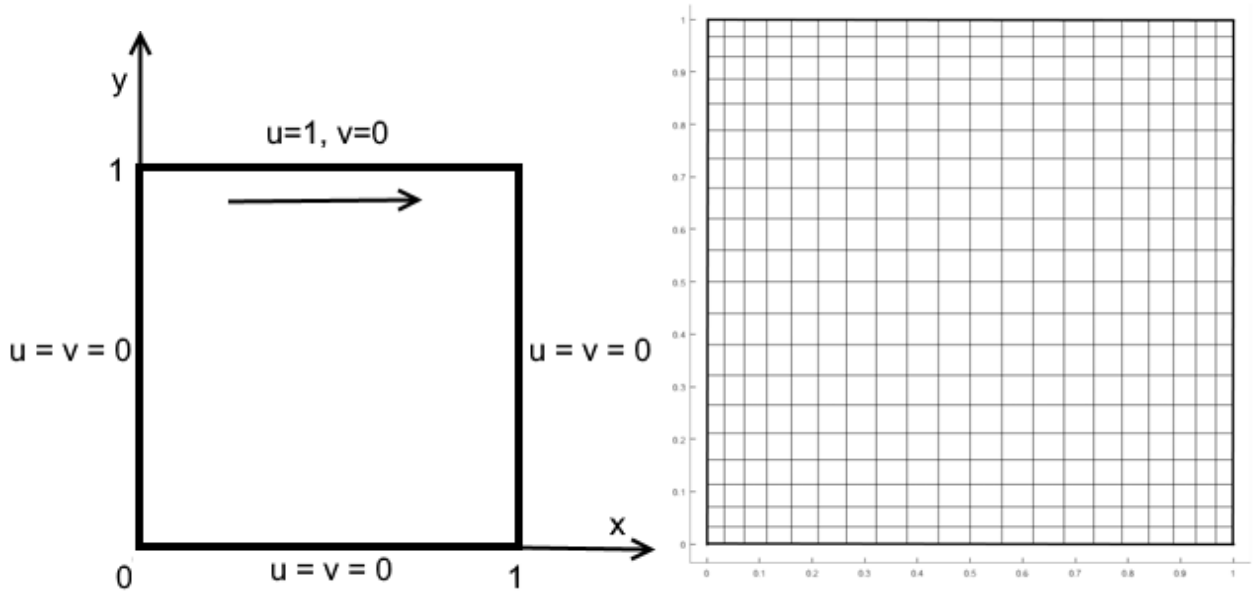

Figure 3: The lid-driven cavity problem geometry, boundary conditions, and computational domains distribution.
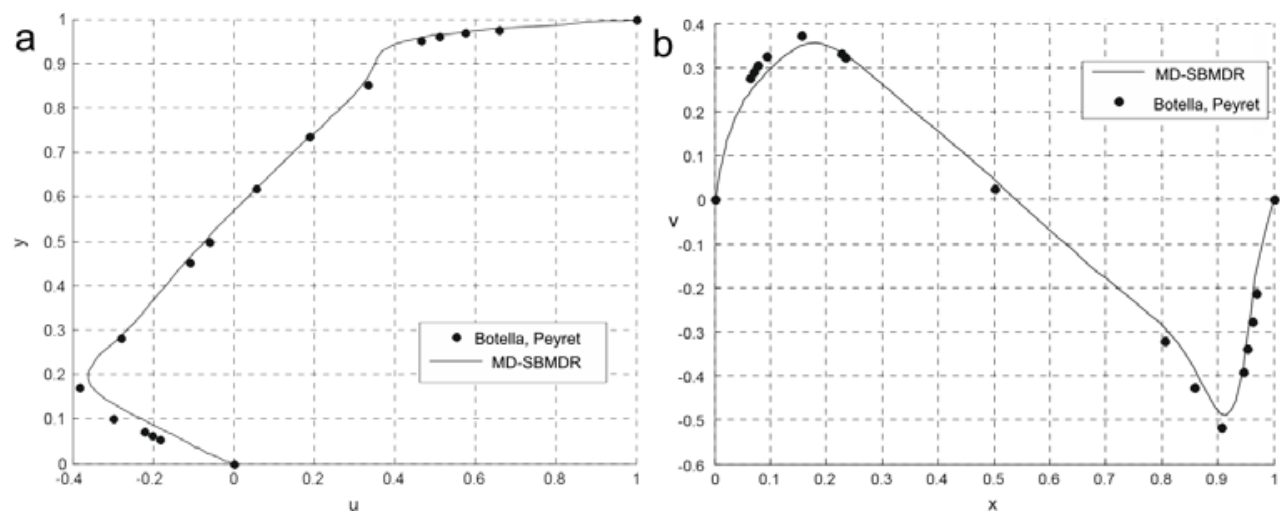

Figure 4: Lid-driven cavity, $\mathrm{Re}=1000$. (a) Horizontal velocity components along vertical line $\mathrm{x}=0.5$; (b) Vertical velocity components along horizontal line $\mathrm{y}=0.5$.

\section{SIMULATION OF THE BACKWARD-FACING STEP FLOW}

The backward-facing step flow is another benchmark problem widely used to test the efficiency of the incompressible flow solver. Numerous numerical and experimental simulations of backward-facing step were performed [16]-[18]. The inlet velocity boundary condition is prescribed as fully developed flow, and maximum inflow velocity is $u_{\max }=1.5$, and the average inflow velocity is $u_{a}=1$. The Reynolds number can then be defined as

$$
R e=\frac{u_{a} H}{v},
$$

where $H$ is the height of the channel (see Fig. 7). 

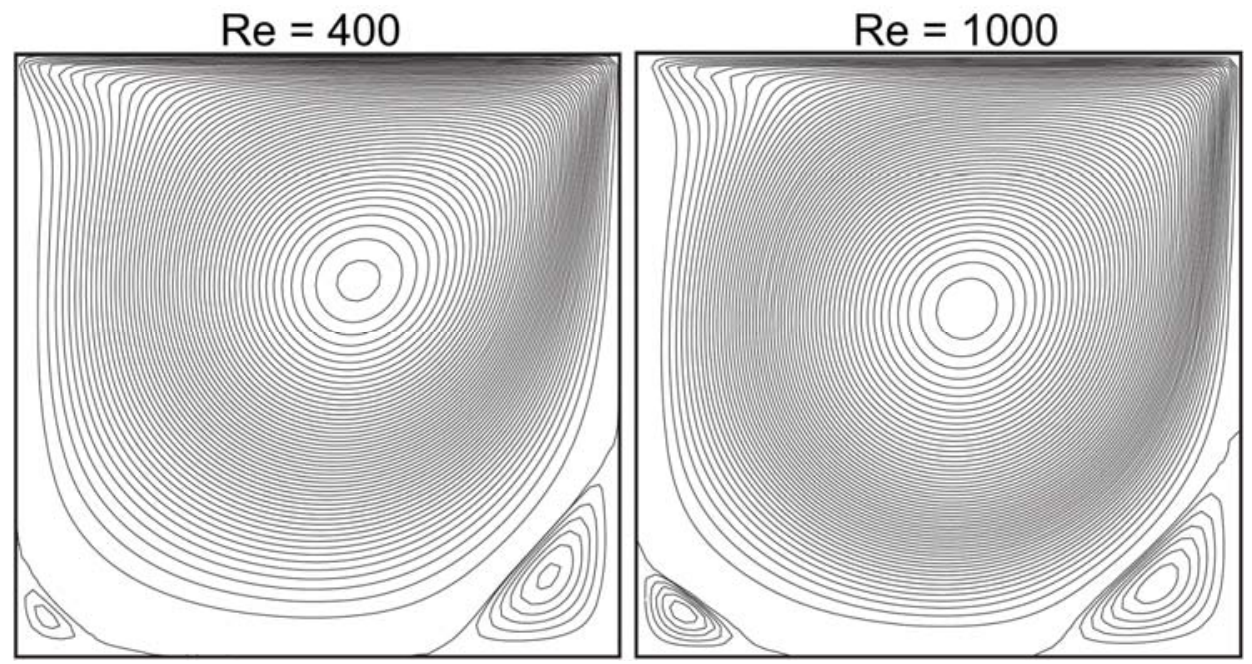

Figure 5: Stream function contours (streamlines) for $\mathrm{Re}=400$ and 1000.
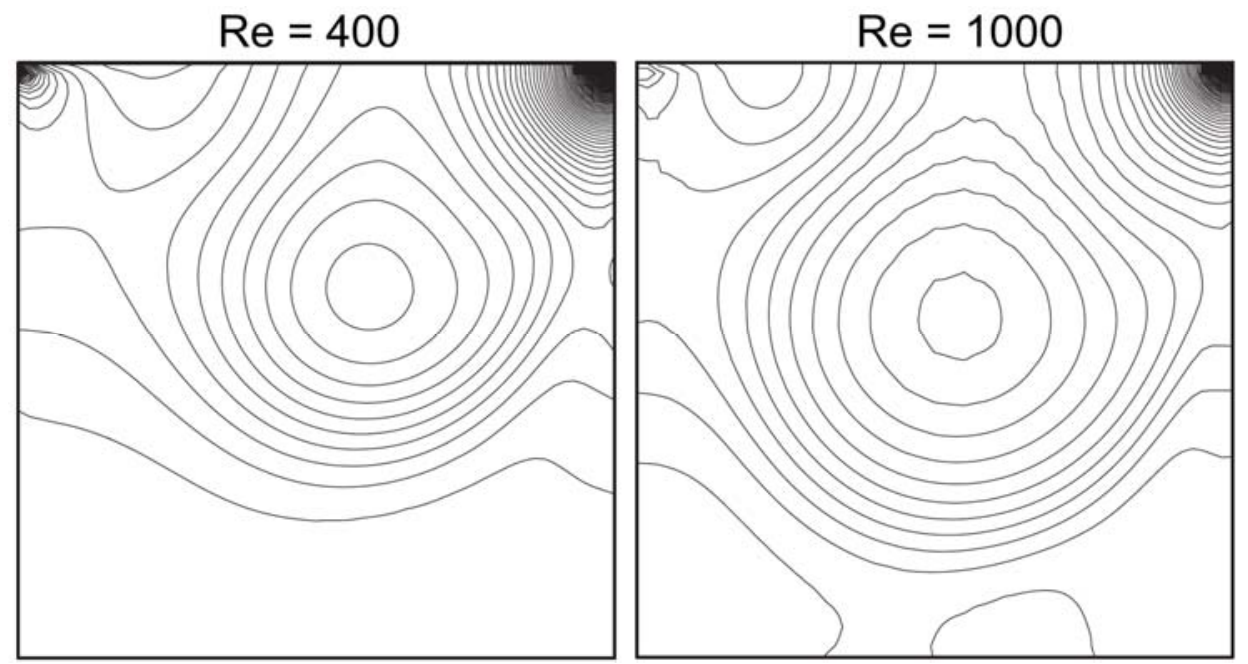

Figure 6: Pressure contours for $\mathrm{Re}=400$ and 1000 .

Stream function boundary conditions for a backward-facing step can be expressed in the following manner:

$$
\begin{gathered}
\psi(x, 0)=0 x \in[0, L], \\
\psi(0, y)=0 y \in[0,0.5], \\
\psi(0, y)=2 y^{2}(3-4 y) y \in[0.5,1], \\
\psi(L, y)=0.25+\frac{\left(3-4 y^{2}\right) y}{4} y \in[0,1], \\
\psi(x, 1)=0 x \in[0, L] .
\end{gathered}
$$




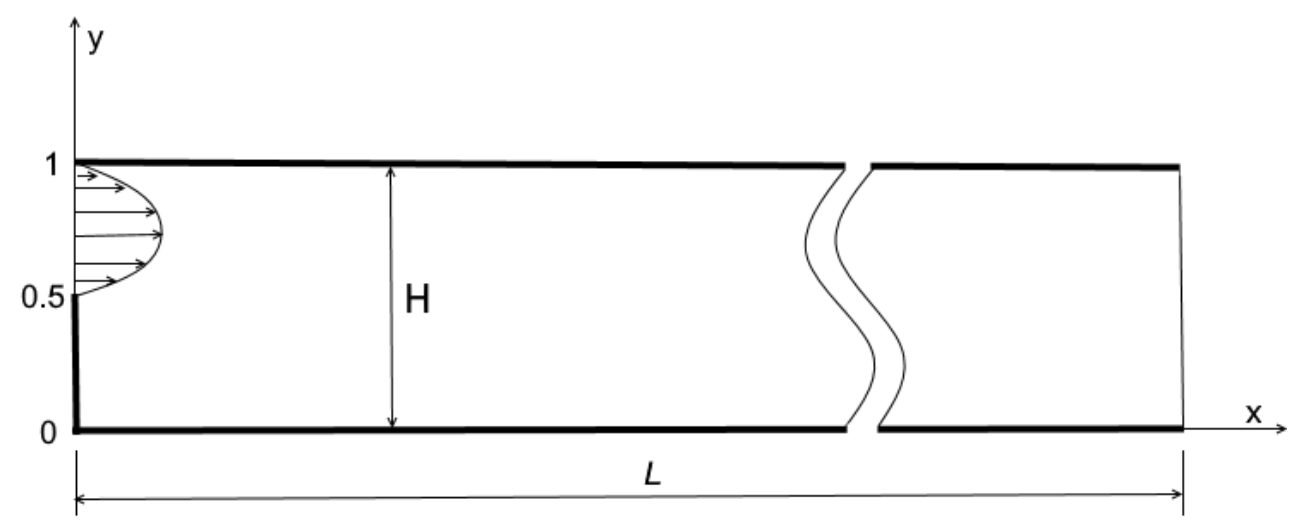

Figure 7: The geometry of the backward-facing step problem.
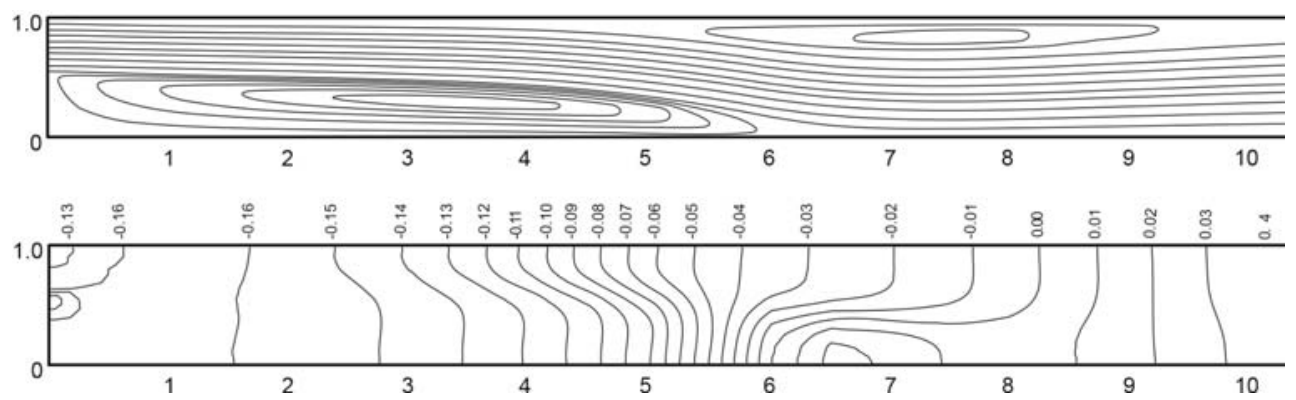

Figure 8: Stream function contours (streamlines) and pressure contours for backwardfacing step, $\mathrm{Re}=800$.
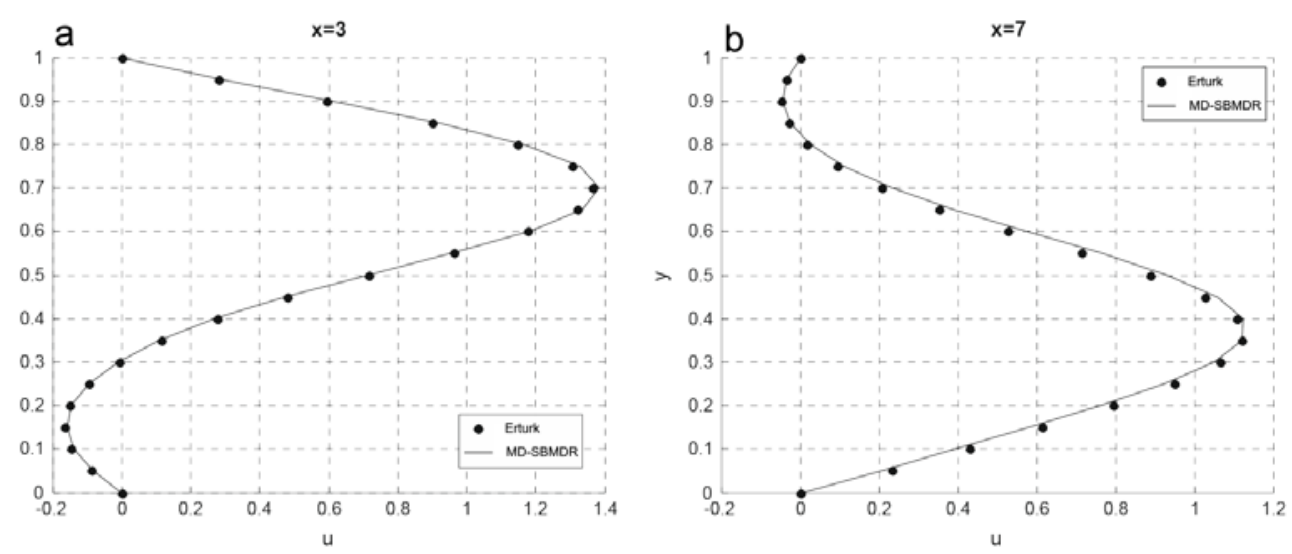

Figure 9: Backward-facing step, comparison of the horizontal velocities in profiles $x=3$ and $\mathrm{x}=7$ for $\mathrm{Re}=800$. 
The computational domain of this problem is covered by uniform cell mesh that is made up of 161 cells in the horizontal and 11 in the vertical direction. The steady solution is reached when the difference between two consecutive stream function solutions is lower than a prescribed tolerance.

Fig. 8 shows streamlines and pressure contours for $\mathrm{Re}=800$, and Fig. 9 shows the comparison of horizontal velocity values in vertical cuts positioned at $x=3$ and $x=7$, with the solution presented in [18]. Comparing results of the MD-SBMDR for $\mathrm{Re}=800$ with results of other researchers is highly identical.

\section{CONCLUSIONS}

This article presents the application of multi-domain SBM and DRM (MD-SBMDR) for the solution the incompressible laminar flow described by a biharmonic stream-velocity form of Navier-Stokes equation. Two types of benchmarking problems, lid-driven cavity and backward-facing step, have been successfully tested. This type of boundary collocation method showed a potential to be a promising alternative to the solutions of incompressible fluid flow using the boundary based numerical schemes. The presented numerical code and numerical results need to be more analyzed in the sense of precision, convergence and computational demands.

\section{ACKNOWLEDGEMENT}

This contribution is the result of the project funded by the Scientific Grant Agency of Slovak Republic (VEGA) No. 1-0716-17.

\section{REFERENCES}

[1] Brebbia, C.A., Telles, J.C.F. \& Wrobel, L.C., Boundary Element Techniques, Springer-Verlag: Berlin and New York, 1984.

[2] Partridge, P.W., Brebbia, C.A. \& Wrobel, L.C., The Dual Reciprocity Boundary Element Method, CM Publications: Southampton, 1992.

[3] Golberg, M., The method of fundamental solutions for Poissons equations. Engineering Analysis with Boundary Elements, 16, pp. 205-213, 1995.

[4] Golberg, M.A. \& Chen, C.S., The method of fundamental solutions for potential. Helmholtz and Diffusion Problems, ed. M. A. Golberg, Boundary Integral MethodsNumerical and Mathematical Aspects, Computational Mechanics Publication: Southampton, pp. 103-176, 1998.

[5] Sarler, B., Solution of potential flow problems by the modified method of fundamental solutions: Formulations with the single layer and the double layer fundamental solutions. Engineering Analysis with Boundary Elements, 33(12), pp. 1374-1382, 2009. DOI: 10.1016/j.enganabound.2009.06.008.

[6] Chen, W., Fu, Z. \& Wei, X., Potential problems by singular boundary method satisfying moment condition. CMES-Computer Modeling in Engineering and Sciences, 54, pp. 65-85, 2009.

[7] Chen, W. \& Gu, Y., Recent advances on singular boundary method. Joint International Workshop for Trefftz method, 4, pp. 543-558.

[8] Fu, Z.J., Chen, W., Chen, J.T. \& Qu, W.Z., Singular boundary method: Three regularization approaches and exterior wave applications. CMES-Computer Modeling in Engineering and Sciences, 99, pp. 417-443, 2014.

[9] Wei, X., Chen, W., Sun, L. \& Chen, B., A simple accurate formula evaluating origin intensity factor in singular boundary method for two-dimensional potential problems 
with Dirichlet boundary. Engineering Analysis with Boundary Elements, 58, pp. 151165, 2015. DOI: 10.1016/j.enganabound.2015.04.010.

[10] Kim, Y., Kim, D., Jun, S. \& Lee, J., Meshfree point collocation method for the streamvorticity formulation of 2D incompressible Navier-Stokes equations. Computer Methods in Applied Mechanics and Engineering, 196(33-34), pp. 3095-3109, 2007. DOI: 10.1016/j.cma.2007.01.018.

[11] Gupta, M. \& Kalita, J., A new paradigm for solving Navier-Stokes equations: streamfunction-velocity formulation. Journal of Computational Physics, 207(1), pp. 52-68, 2005. DOI: 10.1016/j.jcp.2005.01.002.

[12] Yang, C. \& Li, X., Meshless singular boundary methods for biharmonic problems. Engineering Analysis with Boundary Elements, 56, pp. 39-48, 2015.

DOI: 10.1016/j.enganabound.2015.02.001.

[13] Kováŕík, K., Mužík, J., Masarovičová, S. \& Sitányiová, D., Regularized singular boundary method for 3D potential flow. Engineering Analysis with Boundary Elements, 95, pp. 85-92, 2018. DOI: 10.1016/j.enganabound.2018.06.022.

[14] Florez, W., Nonlinear flow using dual reciprocity. WIT Press: Southhampton, UK, 2001.

[15] Botella, O. \& Peyret, R., Benchmark spectral results on the lid-driven cavity flow. Computers and Fluids, 27(4), pp. 421-433, 1998.

DOI: 10.1016/s0045-7930(98)00002-4.

[16] Armaly, B.F., Dursts, F., Pereira, J.C.F. \& Schonung, B., Experimental and theoretical investigation of backward-facing step flow. Journal of Fluid Mechanics, 127(1), pp. 473-496, 1983. DOI: 10.1017/s0022112083002839.

[17] Gartling, D.K., A test problem for outflow boundary conditions-flow over backwardfacing step. International Journal for Numerical Methods in Fluids, 11(7), pp. 953967. DOI: 10.1002/fld.1650110704.

[18] Erturk, E., Numerical solutions of 2-D steady incompressible flow over a backwardfacing step, Part I: high Reynolds number solutions. Computers and Fluids, 37(6), pp. 633-655, 2008. DOI: 10.1016/j.compfluid.2007.09.003. 